Article

\title{
Crystallographic Orientation Influence on the Serrated Yielding Behavior of a Single-Crystal Superalloy
}

\author{
Mikael Segersäll $^{1, *}$ and Johan J. Moverare ${ }^{1,2}$ \\ 1 Department of Management and Engineering, Linköping University, Linköping 58183, Sweden; \\ E-Mail: johan.moverare@liu.se \\ 2 Siemens Industrial Turbomachinery AB, Finspång 61283, Sweden \\ * Author to whom correspondence should be addressed; E-Mail: mikael.segersall@liu.se; \\ Tel.: +46-13-281-145; Fax: +46-13-282-505.
}

Received: 9 November 2012; in revised form: 10 January 2013 / Accepted: 29 January 2013 / Published: 31 January 2013

\begin{abstract}
Since Ni-based single-crystal superalloys are anisotropic materials, their behavior in different crystal orientations is of great interest. In this study, the yielding behavior in both tension and compression for $\langle 001\rangle,\langle 011\rangle$ and $\langle 111\rangle$ oriented materials at $500{ }^{\circ} \mathrm{C}$ has been investigated. The $\langle 011\rangle$ direction showed a serrated yielding behavior, a great tension/compression asymmetry in yield strength and visible deformation bands. However, the $\langle 001\rangle$ and $\langle 111\rangle$ directions showed a more homogeneous yielding, less tension/compression asymmetry in yield strength and no deformation bands. Microstructure investigations showed that the serrated yielding behavior of the $\langle 011\rangle$ direction can be attributed to the appearance of dynamic strain aging (DSA) and that only one slip system is active in this direction during plastic deformation.
\end{abstract}

Keywords: single-crystals; superalloy; yield phenomena; tension/compression asymmetry; dynamic strain aging

\section{Introduction}

Ni-based single-crystal superalloys are used as blade material in gas turbines and aero engines thanks to their excellent resistance to mechanical and chemical degradation at elevated temperatures [1,2]. By using single-crystal instead of poly-crystal material in gas turbine blades, both fatigue and creep properties are enhanced. Commonly, the $\langle 001\rangle$ direction is aligned along the length 
of the turbine blades, since this direction has the lowest Young's modulus of the $\langle 001\rangle,\langle 011\rangle$ and $\langle 111\rangle$ directions, and therefore best fatigue properties. However, the other main directions, $\langle 011\rangle$ and $\langle 111\rangle$, are also of great interest since crack propagation in turbine blades sometimes is dependent on the properties in these directions.

It is well recognized that Ni-based single-crystal superalloys exhibit a number of surprising mechanical properties, including an increase in yield strength with temperature, strong orientation dependence of the yield stress and a tension/compression asymmetry [3-6]. Traditionally the dependence of the flow stress on both temperature and crystallographic orientation has been attributed to the cross-slip of dislocations from the octahedral planes $\{111\}$, to the cube planes $\{100\}$, in the $\gamma^{\prime}$-precipitates [7,8]. However, according to Lall et al. [9], the cross-slip is not only aided by the resolved shear stress on the $\{100\}$ cross-slip plane, but also by the stress tending to constrict the $\mathrm{a} / 6\langle 112\rangle$ Shockley partial dislocations on the primary glide plane, often referred to as "the core width effect". This is believed to be the main reason for the tension/compression asymmetry at low and intermediate temperatures. Later on, more advanced models were derived assuming that deformation after cross-slip can occur by the lateral motion of superkink segments, lying on the $\{111\}$ planes, which links the Kear-Wilsdorf locks that were formed on the $\{010\}$ planes during cross-slip [10,11].

Sometimes also a serrated yielding behavior is in progress during plastic deformation. Many metallic materials show a serrated flow, also known as the Portevin-Le Chatelier (PLC) effect. The reason for this behavior is the interaction between diffusing solute atoms and moving dislocations, more commonly known as dynamic strain aging (DSA). It is well known that the DSA phenomena may lead to a serrated yielding effect and in the literature one can find that DSA occur in superalloys at temperatures from 260 to $800{ }^{\circ} \mathrm{C}$ [12]. Research has showed that also interaction of planar slip bands can cause serrated yielding behavior [13]. Other literature [14] reported a plastic deformation inhomogeneity in different crystallographic directions for the DD8 single-crystal superalloy. In compression at $550{ }^{\circ} \mathrm{C}$, the $\langle 110\rangle$ direction showed a stronger inhomogeneity compared to $\langle 001\rangle$ and $\langle 111\rangle$. The inhomogeneity for the $\langle 110\rangle$ direction was explained by dislocation shearing of $\gamma^{\prime}$-particles, while for the most stable directions, $\langle 001\rangle$ and $\langle 111\rangle$, the deformation process rather was controlled by Orowan by-passing. Miner et al. [15] reported a serrated yielding behavior followed by loud pops for the $\langle 011\rangle$ direction when loading in tension at $760{ }^{\circ} \mathrm{C}$. This was explained by the fact that only the primary octahedral slip system,(111)[101], was active for the $\langle 011\rangle$ direction while several octahedral slip systems were active for the $\langle 001\rangle$ direction.

Studies concerning the behavior of $\langle 001\rangle$ oriented single-crystal superalloys are quite commonly found in the literature. However, comparisons of behavior between different crystal orientations are more rare. The purpose of this paper is therefore to investigate the differences in intermediate temperature yielding behavior, both in tension and compression, between the three crystallographic directions, $\langle 001\rangle,\langle 011\rangle$ and $\langle 111\rangle$.

\section{Experimental Procedure}

In this study the Ni-based single-crystal superalloy MD2 with chemical composition $\mathrm{Ni}, 5.1 \mathrm{Co}$, 6.0 Ta, 8.0 Cr, 8.1 W, 5.0 Al, 1.3 Ti, 2.1 Mo, 0.1 Hf and 0.1 Si was investigated. The material was solution heat treated at $1275{ }^{\circ} \mathrm{C}$ for $8 \mathrm{~h}$ followed by a two-stage aging process with $3 \mathrm{~h}$ at $1100{ }^{\circ} \mathrm{C}$ and 
$24 \mathrm{~h}$ at $850{ }^{\circ} \mathrm{C}$. Test specimens were machined from cast bars and the deviation from the ideal orientation was less than $2^{\circ}$ for the $\langle 011\rangle$ and $\langle 111\rangle$ specimens, but varied between $6^{\circ}$ and $12^{\circ}$ for the $\langle 001\rangle$ specimens. The stress-strain response during monotonic tensile and compressive loading was investigated using a servohydraulic testing machine. The tests were conducted at $500{ }^{\circ} \mathrm{C}$ under displacement control, while monitoring strain and load, with an initial strain rate of $\sim 10^{-5} \mathrm{~s}^{-1}$. The samples were carefully aligned to prevent buckling. The tests were interrupted when approximately $1 \%$ plastic strain was achieved, and the specimens were then unloaded, cooled rapidly to room temperature (RT) and removed from the test machine. After the tests, all specimens were investigated by stereomicroscopy before they were cut parallel to the loading direction for further investigation by scanning electron microscopy (SEM) in a Hitachi SU70 SEM. The SEM samples were prepared by grinding and mechanical polishing, but no samples were etched. Acceleration voltages from 10 to $20 \mathrm{kV}$ were used during the SEM investigation. Most images were taken using backscattered electrons and channelling contrast. Electron backscatter diffraction (EBSD) analysis was performed using an Oxford Instrument Nordlys detector.

\section{Results and Discussion}

\subsection{Mechanical Testing}

The results from tensile and compressive tests at $500{ }^{\circ} \mathrm{C}$ for the three crystallographic directions $\langle 001\rangle,\langle 011\rangle$ and $\langle 111\rangle$ are presented in Figure 1.

Figure 1. Tension and compression tests at $500{ }^{\circ} \mathrm{C}$ for $\langle 001\rangle,\langle 011\rangle$ and $\langle 111\rangle$ directions.

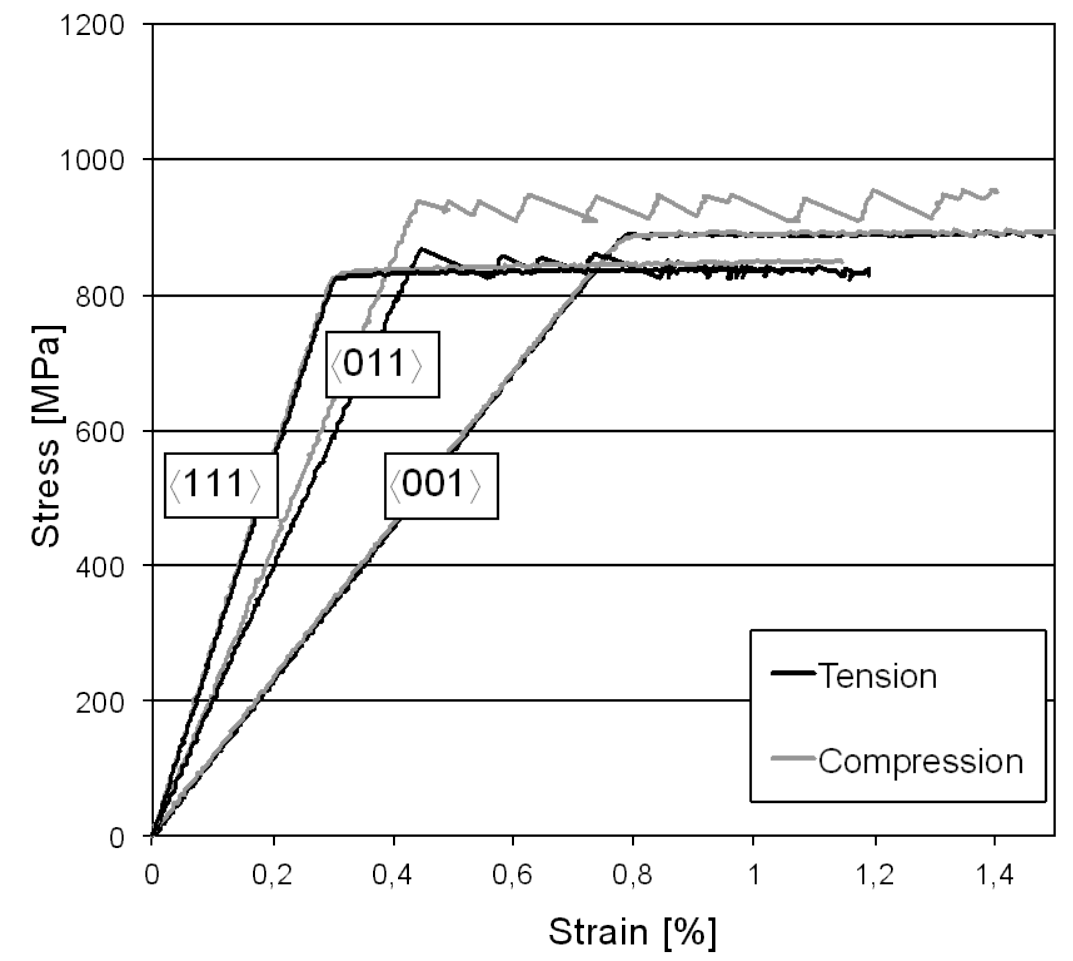

As expected, the $\langle 111\rangle$ direction showed the highest stiffness of all directions, approximately $300 \mathrm{GPa}$ in both tension and compression. The $\langle 011\rangle$ direction showed a higher stiffness in 
compression compared to tension, approximately $220 \mathrm{GPa}$ compared to $210 \mathrm{GPa}$. This difference can be attributed to the deviations from the ideal crystal orientation connected to each specimen. The $\langle 001\rangle$ direction showed the lowest stiffness, approximately $115 \mathrm{GPa}$. Single-crystal superalloys exhibit a yield strength tension/compression asymmetry and in this study greatest asymmetry was found for the $\langle 011\rangle$ direction, where the compressive yield strength was around $70 \mathrm{MPa}$ higher compared to that in tension. However, the $\langle 001\rangle$ and $\langle 111\rangle$ directions instead showed similar yield strengths in tension and compression at this temperature, i.e., no significant asymmetry was found for these directions. In addition to the tension/compression asymmetry, the $\langle 011\rangle$ direction also showed a significant serrated yielding behavior, both in tension and compression. On the other hand, yielding for the $\langle 001\rangle$ and $\langle 111\rangle$ directions was more homogeneous. The highest yield strength was found for the $\langle 011\rangle$ direction in compression, $940 \mathrm{MPa}$, followed by the $\langle 001\rangle$ direction where the yield strengths in tension and compression were approximately $880 \mathrm{MPa}$. In tension, the $\langle 011\rangle$ direction showed a yield strength of $865 \mathrm{MPa}$. The $\langle 111\rangle$ orientation was the weakest of all directions; here the yield strength was approximately $825 \mathrm{MPa}$ in both tension and compression.

The results can be compared to another study [5] where the same tests were conducted, with the same alloy, at RT instead of $500{ }^{\circ} \mathrm{C}$. In Figure 2 the yield strengths for all directions at both temperatures are summarized in one graph.

Figure 2. Yield strengths for $\langle 001\rangle,\langle 011\rangle$ and $\langle 111\rangle$ directions at RT and $500{ }^{\circ} \mathrm{C}$. RT yield strengths are taken from [5].

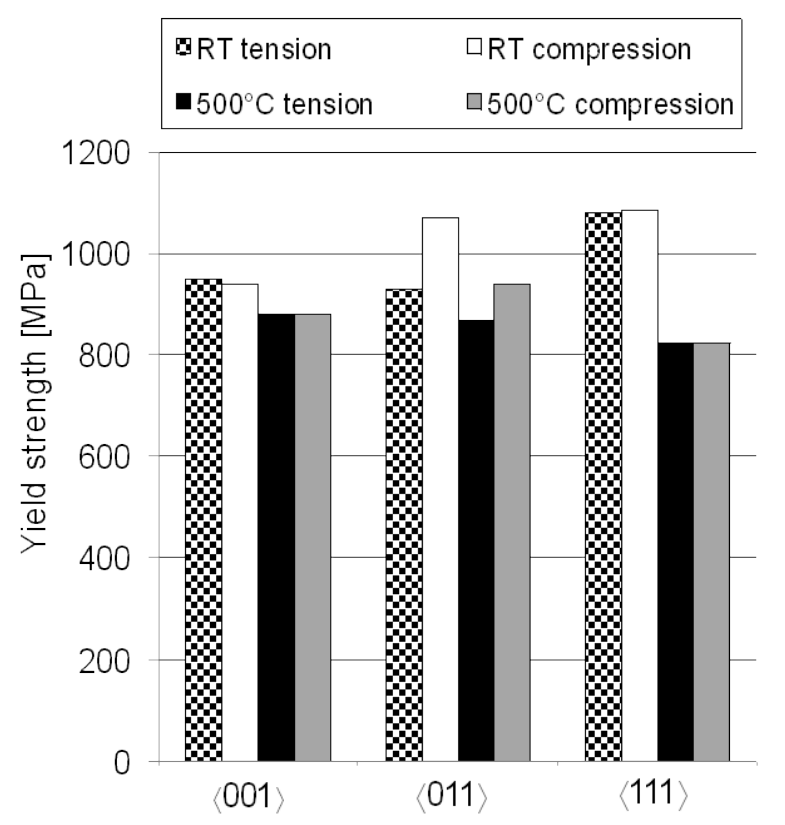

At both temperatures, the greatest tension/compression asymmetry in yield strength was found for the $\langle 011\rangle$ direction. However, at RT no serrated yielding behavior was found for the $\langle 011\rangle$ direction. This indicates that serrated yielding depends on diffusion and DSA. Further comparison to that study shows that the $\langle 111\rangle$ direction had similar or even higher yield strength compared to the other directions at RT but at $500{ }^{\circ} \mathrm{C}$ this direction is the weakest. The $\langle 111\rangle$ direction therefore show the biggest reduction in yield strength, approximately $25 \%$ when going from RT to $500{ }^{\circ} \mathrm{C}$. For the $\langle 001\rangle$ 
direction the same comparison shows a reduction in yield strength of around 7\% only. This assumes that the yield strengths in tension and compression are approximately the same for those directions. When looking at the $\langle 011\rangle$ direction, where the greatest tension/compression asymmetry was found, the tensile yield strength is $7 \%$ lower at $500{ }^{\circ} \mathrm{C}$ compared to RT while the compressive yield strength decreases $12 \%$ when going from RT to $500{ }^{\circ} \mathrm{C}$.

\subsection{Microscopy}

Specimen investigation by stereomicroscopy showed clear crystallographic deformation bands stretching over the $\langle 011\rangle$ specimen surfaces, both in tension and compression. However, for $\langle 001\rangle$ and $\langle 111\rangle$ oriented specimens no clear deformation bands were visible on the surfaces. Unfortunately, since no specimens were polished prior to testing, it was very difficult to obtain high quality images of the deformation bands appearing on the $\langle 011\rangle$ oriented specimen surfaces, and therefore only a sketch showing the deformation band appearance is shown in Figure 3. The slip traces on the $\langle 011\rangle$ oriented specimens are consistent with slip along the (111)[101] slip system.

Figure 3. A sketch of the deformation band appearance on the specimen surfaces.

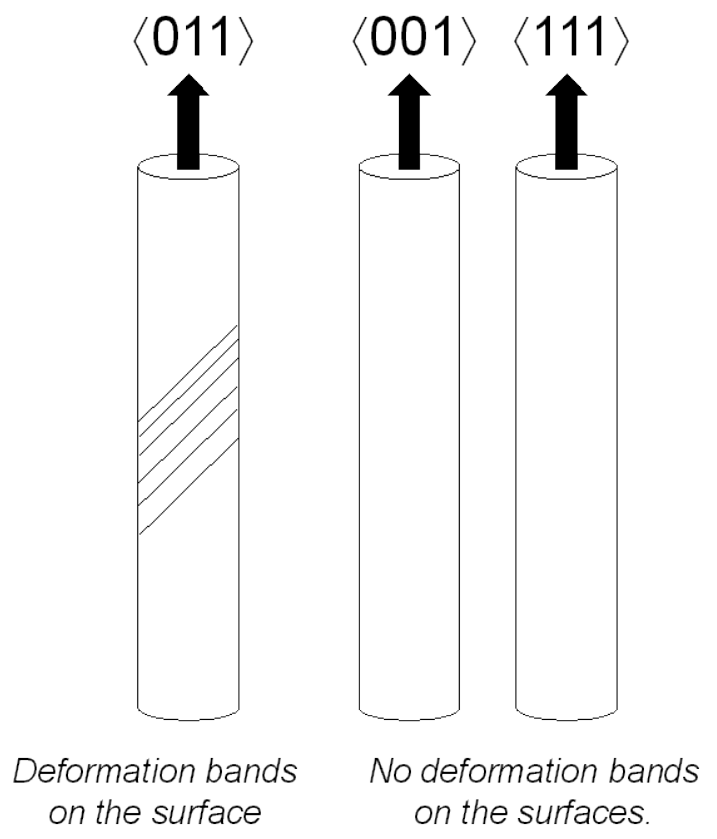

Further investigation by SEM, using backscattered electrons, also showed clear deformation bands in the microstructure for the $\langle 011\rangle$ specimens. Figure 4a shows the typical appearance of deformation bands in the $\gamma / \gamma^{\prime}$-microstructure at low magnification for the $\langle 011\rangle$ oriented specimen subjected to compressive loads. Investigations of the same deformation band at a higher magnification did not reveal any significant shearing of the $\gamma / \gamma^{\prime}$-microstructure, Figure $4 \mathrm{~b}$. This is probably due to the fact that the tests were interrupted after $1 \%$ plastic deformation. Further deformation probably would have revealed dislocations cutting of the $\gamma^{\prime}$-phase.

EBSD was performed to further examine the orientation variations between the deformation bands and the less deformed $\gamma / \gamma^{\prime}$-microstructure. The mapping showed very small orientation variations, up to $1^{\circ}$, between the deformation bands and the less deformed microstructure. This means that twinning 
of the microstructure is not present within the deformation bands. In Figure 4c an EBSD map of such a deformation band is shown where the white segments indicate orientation differences from $0.5^{\circ}$ to $1^{\circ}$. Interestingly, it is clear that the orientation differences (see white segements in Figure 4c) are concentrated outside the deformation band, while the band itself contains much less orientation differences. The orientation differences outside the band are very likely coupled to the misorientation between the $\gamma$ - and $\gamma^{\prime}$-phases. Inside the band, the $\gamma / \gamma^{\prime}$-misorientation has probably been equalised by dislocations cutting the $\gamma^{\prime}$-phase, resulting in less orientation differences. The deformation bands are therefore very likely bundles of glide bands, i.e., dislocations that propagate through the crystal. Hence, the dislocation density within the deformation bands is higher compared to the less deformed microstructure. Deformation bands in $\langle 011\rangle$ oriented material has been reported before in the literature [16], and in that study the deformation bands were detected as changes in $\gamma$-channel size within the microstructure. This seems not to be the case in this study.

Figure 4. The $\langle 011\rangle$ specimen subjected to compressive stress; (a) a backscattered electron image of deformation bands at 1200 times magnification; (b) same deformation band as in (a) at 7000 times magnification; (c) EBSD map of such a deformation band, where each white segment corresponds to an orientation difference of $0.5^{\circ}-1^{\circ}$.
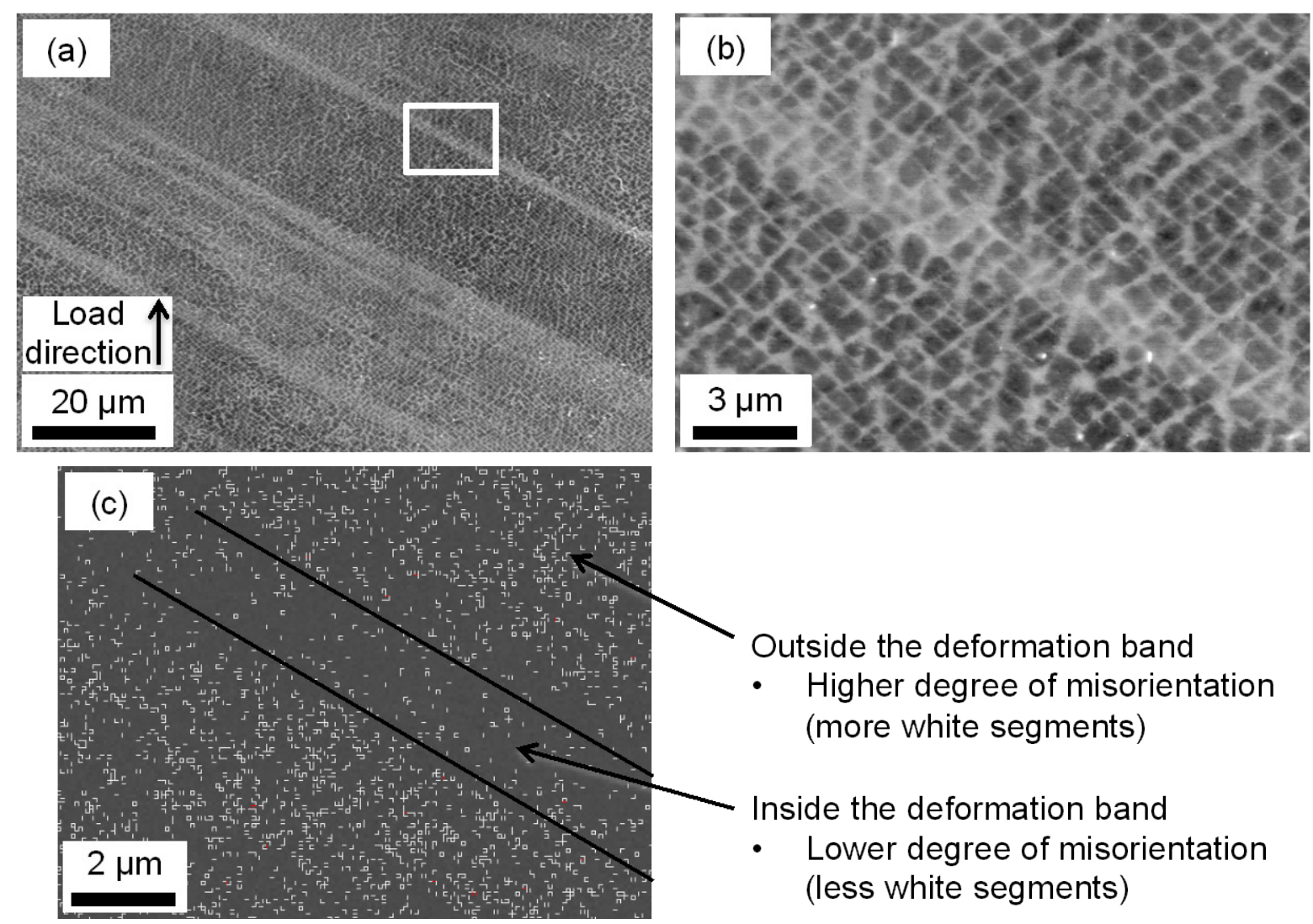

Thus, the $\langle 011\rangle$ direction shows deformation bands, which are bundles of glide bands, and a serrated yielding behavior while the other directions show stable yielding and no deformation bands. A serrated yielding behavior is often explained by DSA and this has very likely occurred within the glide bands found in the $\langle 011\rangle$ oriented material. DSA is a phenomenon where moving dislocations are pinned by diffusing solute atoms and this is it more probable to occur in regions with a high 
dislocation density, i.e., the deformation bands. This can explain the serrated yielding behavior showed by the $\langle 011\rangle$ direction. The $\langle 001\rangle$ and $\langle 111\rangle$ directions showed no serrated yielding behavior and neither any glide bands in the microstructure. With no glide bands, the potential for DSA to occur is smaller compared to when glide bands are present. Once again comparing to the previous study [5], no serrated yielding effect was found in any direction when testing at RT. This is reasonable since DSA is a diffusion dependent process, and the interaction between solute atoms and moving dislocations are more likely to occur at $500{ }^{\circ} \mathrm{C}$ compared to RT.

Hence, DSA in combination with highly localized deformation bands is one reason for the serrated yielding behavior of the $\langle 011\rangle$ direction. This is in line with previous research by Miner et al. [14], who concluded that only one slip system is active during yielding for the $\langle 011\rangle$ direction while multiple slip systems are active in the other directions. Due to symmetry reasons, the $\langle 011\rangle$ direction has 4 slip systems with the same Schmid factor, while the $\langle 001\rangle$ direction has 8 and the $\langle 111\rangle$ direction has 6 equivalent slip systems. However, since each specimen is not aligned exactly in the $\langle 011\rangle$ direction, there will be one slip system that have a higher resolved shear stress and will operate as the primary slip system. Due to lower symmetry (less equivalent slip systems) of the $\langle 011\rangle$ direction compared to the $\langle 001\rangle$ and $\langle 111\rangle$ directions, the $\langle 011\rangle$ direction has a higher tendancy for single slip compared to the other orientations. When multiple slip systems are active, there will always be one system where dislocation glide can occur during plastic deformation and the yielding becomes more stable. However, when only one slip system is active, the dislocations in the glide bands can only propagate in one direction and are therefore sometimes stopped, if the stress is not big enough, before they can propagate any further. This leads to the serrated yielding effect. With only one slip system active, it is also more likely to obtain those concentrated glide bands which end up as topographic differences on the specimen surface. For the $\langle 001\rangle$ and $\langle 111\rangle$ directions, when multiple slip systems were active at the same time the surface becomes more homogenous with less topographic difference.

\section{Conclusions}

To conclude this study, it is very clear that the $\langle 011\rangle$ direction demonstrated a tension/compression asymmetry and a serrated yielding behavior in both tension and compression, at $500{ }^{\circ} \mathrm{C}$. For this direction, deformation bands were also found both on the surface and in the $\gamma / \gamma^{\prime}$-microstructure, and the deformation bands are very likely bundles of glide bands. The main reason for the serrated yielding behavior of the the $\langle 011\rangle$ direction is the occurrence of DSA in combination with highly localized deformation bands. In contrast to this, the $\langle 001\rangle$ and $\langle 111\rangle$ directions showed a more homogeneous yielding behavior, no distinct deformation bands and no tension/compression asymmetry.

\section{Acknowledgments}

The work has been financially supported by Siemens Industrial Turbomachinery AB in Finspång, Sweden and the Swedish Energy Agency, via the Research Consortium of Materials Technology for Thermal Energy Processes, Grant No. KME-502. 


\section{References}

1. Reed, R.C.; Tao, T.; Warnken, N. Alloys-by-design: Application to nickel-based single crystal superalloys. Acta Mater. 2009, 57, 5898-5913.

2. Pollock, T.M.; Tin, S. Nickel-based superalloys for advanced turbine engines: Chemistry, microstructure, and properties. J. Propuls. Power 2006, 22, 361-374.

3. Ezz, S.S.; Pope, D.P.; Paidar, V. The tension/compression flow stress asymmetry in $\mathrm{Ni}_{3}(\mathrm{Al}, \mathrm{Nb})$ single crystals. Acta Metall. 1982, 30, 921-926.

4. Miner, R.V.; Gabb, T.P.; Gayda, J. Orientation and temperature dependence of some mechanical properties of the single-crystal nickel-base superalloy René N4 III-Tension-compression anisotropy. Metall. Trans. A 1986, 17, 507-512.

5. Leidermark, D.; Moverare, J.J.; Simonsson, K.; Sjöström, S.; Johansson, S. Room temperature yield behaviour of a single-crystal nickel-base superalloy with tension/compression asymmetry. Comput. Mater. Sci. 2009, 47, 366-372.

6. Leidermark, D.; Moverare, J.J.; Johansson, S.; Simonsson, K.; Sjöström, S. Tension/compression asymmetry of a single-crystal superalloy in virgin and degraded condition. Acta Mater. 2010, 58, 4986-4997.

7. Takeuchi, S.; Kuramoto, E. Temperature and orientation dependence of the yield stress in $\mathrm{Ni}_{3} \mathrm{Ga}$ single crystals. Acta Metall. 1973, 21, 415-425.

8. Paidar, V.; Pope, D.P.; Vitek, V. A theory of the anomalous yield behavior in $\mathrm{L}_{2}$ ordered alloys. Acta Metall. 1984, 32, 435-448.

9. Lall, C.; Chin, S.; Pope, D.P. The orientation and temperature dependence of the yield stress of $\mathrm{Ni}_{3}(\mathrm{Al}, \mathrm{Nb})$ single crystals. Metall. Trans. A 1979, 10, 1323-1332.

10. Hirsch, P.B. A new theory of the anamalous yield stress in $\mathrm{L}_{12}$ alloys. Philis. Mag. A 1992, 65, 569-612.

11. Mills, M.J.; Chrzan, D.C. Dynamical simulation of dislocation motion in $\mathrm{L}_{12}$ alloys. Acta Metall. Mater. 1992, 40, 3051-3064.

12. Sengupta, A.; Putatunda, S.K. Influence of dynamic strain aging on the near-threshold fatigue crack growth behavior of a new single crystal nickel-based superalloy. Scr. Metall. Mater. 1994, 31, 1163-1168.

13. Rong, T.S. Serrated yielding in the B2-ordered Nb-15Al-20V alloy. Intermetallics 2003, 11, 151-155.

14. Wu, X.; Zhang, J.H.; Liu, J.L.; Jin, T.; Xu, Y.B.; Hu, Z.Q. Plastic deformation inhomogeneity in a single crystal nickel-base superalloy. Mater. Sci. Eng. A 2002, 325, 478-483.

15. Miner, R.V.; Voigt, R.C.; Gayda, J.; Gabb, T.P. Orientation and temperature dependence of some mechanical properties of the single-crystal nickel-base superalloy René N4 Part I: Tensile behavior. Metall. Trans. A 1986, 17, 491-496.

16. Westbrooke, E.F.; Forero, L.E.; Ebrahimi, F. Slip analysis in a Ni-base superalloy. Acta Mater. 2005, 53, 2137-2147.

(C) 2013 by the authors; licensee MDPI, Basel, Switzerland. This article is an open access article distributed under the terms and conditions of the Creative Commons Attribution license (http://creativecommons.org/licenses/by/3.0/). 